Document downloaded from:

http://hdl.handle.net/10251/155855

This paper must be cited as:

Guirao Sánchez, AJ.; Martínez-Cervantes, G.; Rodríguez Ruiz, J. (2019). Completeness in the Mackey topology by norming subspaces. Journal of Mathematical Analysis and Applications. 478(2):776-789. https://doi.org/10.1016/j.jmaa.2019.05.054

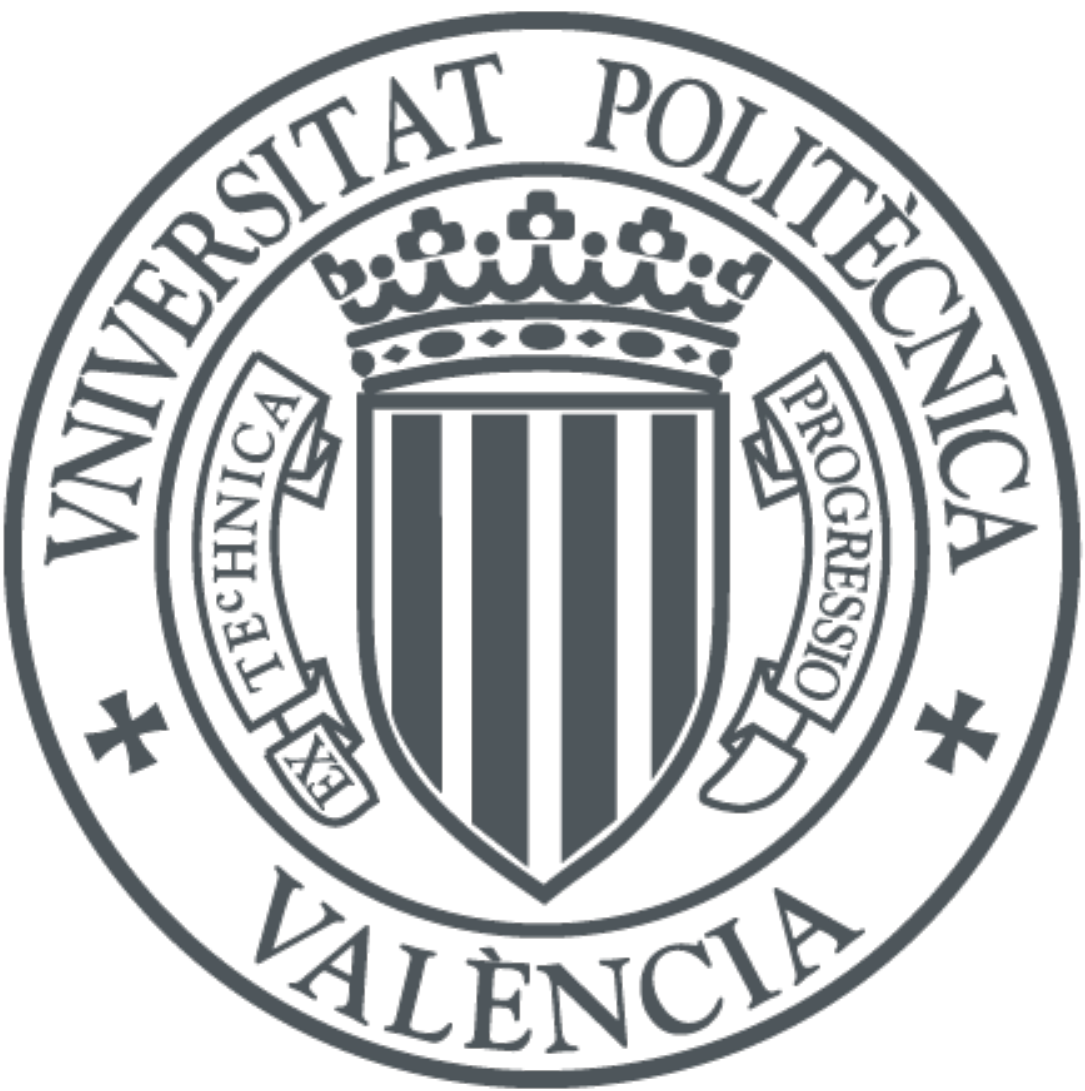

The final publication is available at

https://doi.org/10.1016/j.jmaa.2019.05.054

Copyright Elsevier

Additional Information 


\title{
COMPLETENESS IN THE MACKEY TOPOLOGY BY NORMING SUBSPACES
}

\author{
A.J. GUIRAO, G. MARTÍNEZ-CERVANTES, AND J. RODRÍGUEZ \\ Dedicated to the memory of Bernardo Cascales
}

\begin{abstract}
We study the class of Banach spaces $X$ such that the locally convex space $(X, \mu(X, Y))$ is complete for every norming and norm-closed subspace $Y \subset X^{*}$, where $\mu(X, Y)$ denotes the Mackey topology on $X$ associated to the dual pair $\langle X, Y\rangle$. Such Banach spaces are called fully Mackey complete. We show that fully Mackey completeness is implied by Efremov's property $(\mathcal{E})$ and, on the other hand, it prevents the existence of subspaces isomorphic to $\ell_{1}\left(\omega_{1}\right)$. This extends previous results by Guirao, Montesinos and Zizler [J. Math. Anal. Appl. 445 (2017), 944-952] and Bonet and Cascales [Bull. Aust. Math. Soc. 81 (2010), 409-413]. Further examples of Banach spaces which are not fully Mackey complete are exhibited, like $C\left[0, \omega_{1}\right]$ and the long James space $J\left(\omega_{1}\right)$. Finally, by assuming the Continuum Hypothesis, we construct a Banach space with $w^{*}$-sequential dual unit ball which is not fully Mackey complete. A key role in our discussion is played by the (at least formally) smaller class of Banach spaces $X$ such that $\left(Y, w^{*}\right)$ has the Mazur property for every norming and norm-closed subspace $Y \subset X^{*}$.
\end{abstract}

\section{INTRODUCTION}

Let $X$ be a Banach space and $Y \subset X^{*}$ a $w^{*}$-dense subspace (not necessarily norm-closed). The Mackey topology $\mu(X, Y)$ on $X$ associated to the dual pair $\langle X, Y\rangle$ is the locally convex topology of uniform convergence on elements of the family

$$
\mathcal{K}(Y):=\left\{K \subset Y: K \text { is absolutely convex and } w^{*} \text {-compact }\right\} .
$$

Several authors have recently discussed the completeness of $(X, \mu(X, Y))$, see $[3$, [8] and [9. This research line was motivated initially by Kunze's paper [1] on vector integration (cf. [1, 15]). Bonet and Cascales [3] exploited some results of [7] to prove that if $X$ contains a subspace isomorphic to $\ell_{1}(\mathfrak{c})$, then there is a norming and norm-closed subspace $Y \subset X^{*}$ for which $(X, \mu(X, Y))$ is not complete. At this point we stress that, in general, the completeness of $(X, \mu(X, Y))$ implies that $Y$ is norming, see [9. Proposition 3]. Guirao, Montesinos and Zizler [9] exhibited a

2010 Mathematics Subject Classification. 46A50, 46B26.

Key words and phrases. Mackey topology; completeness; norming subspace; Mazur property.

A.J. Guirao was supported by projects MTM2017-83262-C2-1-P (AEI/FEDER, UE) and 19368/PI/14 (Fundación Séneca). G. Martínez-Cervantes and J. Rodríguez were supported by projects MTM2014-54182-P and MTM2017-86182-P (AEI/FEDER, UE) and 19275/PI/14 (Fundación Séneca). 
connection between the completeness of $(X, \mu(X, Y))$ and the Mazur property of $\left(Y, w^{*}\right)$ (i.e. the property that every $w^{*}$-sequentially continuous linear functional $f: Y \rightarrow \mathbb{R}$ is $w^{*}$-continuous). More precisely:

(a) If $\left(Y, w^{*}\right)$ has the Mazur property and $Y$ is norm-closed, then $(X, \mu(X, Y))$ is complete, see [9, Proposition 10].

(b) If $(X, \mu(X, Y))$ is complete and every $K \in \mathcal{K}(Y)$ is Fréchet-Urysohn, then $\left(Y, w^{*}\right)$ has the Mazur property, see [9, Proposition 1].

Let us introduce a couple of definitions:

Definition 1.1. A Banach space $X$ is said to be fully Mackey complete (resp. fully Mazur $)$ if $(X, \mu(X, Y))$ is complete (resp. $\left(Y, w^{*}\right)$ has the Mazur property) for every norming and norm-closed subspace $Y \subset X^{*}$.

Thus, statement (a) above implies that every fully Mazur space is fully Mackey complete. A sufficient condition on a Banach space $X$ to be fully Mazur is that $\left(B_{X^{*}}, w^{*}\right)$ is Fréchet-Urysohn (see [9, Theorem 5]), which includes the case of weakly compactly generated spaces and, more generally, weakly Lindelof determined ones. On the other hand, the aforementioned result of [3] says that a fully Mackey complete Banach space cannot contain subspaces isomorphic to $\ell_{1}(\mathfrak{c})$.

In this paper we go a bit further in studying fully Mazur and fully Mackey complete Banach spaces. The paper is organized as follows.

Section 2 introduces the basic terminology and contains some preliminary known results on the completeness of Mackey topologies. In addition, we prove that completeness and quasi-completeness are equivalent for $(X, \mu(X, Y))$ whenever $Y$ is norm-closed (Proposition 2.5).

We begin Section 3 by showing that in statement (b) above it is enough to assume (besides the completeness of $(X, \mu(X, Y)))$ that every convex $w^{*}$-sequentially closed subset of any $K \in \mathcal{K}(Y)$ is $w^{*}$-closed (Proposition 3.1). This is a localization of the Banach space property $\left(\mathcal{E}^{\prime}\right)$ studied in 2, 12, (which means that every convex $w^{*}$-sequentially closed bounded subset of the dual is $w^{*}$-closed). In particular, fully Mackey completeness is equivalent to being fully Mazur for Banach spaces with property $\left(\mathcal{E}^{\prime}\right)$. We stress that property $\left(\mathcal{E}^{\prime}\right)$ is strictly weaker than having Fréchet-Urysohn dual ball, as witnessed by the so-called Johnson-Lindentrauss spaces (see [12, Theorem 3.1]).

Our main results in Section 3 characterize fully Mazur and fully Mackey complete Banach spaces. Write $S_{1}(A) \subset X^{*}$ to denote the set of all limits of $w^{*}$-convergent sequences contained in the set $A \subset X^{*}$. In Theorem 3.3 we prove that a Banach space $X$ is fully Mazur if and only if $S_{1}(Y)=X^{*}$ for every norming and normclosed subspace $Y \subset X^{*}$. As a consequence, every Banach space having Efremov's property $(\mathcal{E})$ is fully Mazur (Corollary 3.4). Recall that $X$ is said to have Efremov's property $(\mathcal{E})$ if $S_{1}(C)=\bar{C}^{w^{*}}$ for every convex bounded set $C \subset X^{*}$ (see [14]). The following implications hold in general:

$\left(B_{X^{*}}, w^{*}\right)$ is Fréchet-Urysohn $\Longrightarrow X$ has property $(\mathcal{E}) \Longrightarrow X$ has property $\left(\mathcal{E}^{\prime}\right)$. Under the Continuum Hypothesis there exist Banach spaces separating the three conditions above (see [2]), while it is unknown whether such examples exist in ZFC. 
On the other hand, in Theorem 3.5 we characterize fully Mackey completeness in a similar spirit, namely, a Banach space $X$ is shown to be fully Mackey complete if and only if for every norming and norm-closed subspace $Y \subset X^{*}$ and every $x^{*} \in X^{*} \backslash Y$ there is $K \in \mathcal{K}\left(X^{*}\right)$ such that $K \subset Y \oplus\left[x^{*}\right]$ and $x^{*} \in \overline{K \cap Y}^{w^{*}}$.

Section 4 is mostly devoted to showing further examples of Banach spaces which are not fully Mackey complete. Theorem 4.1 provides a technical tool which applies to prove that spaces like $\ell_{1}\left(\omega_{1}\right)$ and $C\left[0, \omega_{1}\right]$ fail to be fully Mackey complete. In particular, since this property is inherited by closed subspaces (Corollary 3.6), it follows that a fully Mackey complete Banach space cannot contain subspaces isomorphic to $\ell_{1}\left(\omega_{1}\right)$, thus improving the result of 3 ] which was mentioned above. The absence of subspaces isomorphic to $\ell_{1}$ is not sufficient for fully Mackey completeness, as the example of $C\left[0, \omega_{1}\right]$ makes clear. On the other hand, we also investigate fully Mackey completeness within the setting of dual Banach spaces. It is shown that if $X^{*}$ is fully Mackey complete, then $X$ is $w^{*}$-sequentially dense in $X^{* *}$ (Theorem 4.5). As a consequence, we include a sharp characterization of the fully Mackey completeness of $X^{*}$, in some particular cases, in terms of the compact topological space $\left(B_{X^{* *}}, w^{*}\right)$ (Corollaries 4.8 and 4.9 ).

One may wonder whether property $\left(\mathcal{E}^{\prime}\right)$ implies fully Mackey completeness. We will show that this is not the case. By modifying a construction of [2, under the Continuum Hypothesis, we provide an example of a maximal almost disjoint family $\mathcal{F}$ of infinite subsets of $\mathbb{N}$ for which the Banach space $C\left(K_{\mathcal{F}}\right)$ is not fully Mackey complete, where $K_{\mathcal{F}}$ is the Stone space of the Boolean algebra generated by $\mathcal{F}$ and the finite subsets of $\mathbb{N}$ (Theorem 4.10). Note that, without any extra set-theoretic assumption, all Banach spaces of the form $C\left(K_{\mathcal{F}}\right)$ have property $\left(\mathcal{E}^{\prime}\right)$ (see [12]). We finish the paper by collecting several open problems. For instance, we do not know whether fully Mazur and fully Mackey completeness are equivalent properties.

\section{TeRminology And PRELiminaries}

All our topological spaces are Hausdorff and all our linear spaces are real. Given a linear space $E$, we denote by $E^{\#}$ the linear space consisting of all linear functionals from $E$ to $\mathbb{R}$. For any set $S \subset E$, the symbol $[S]$ stands for the subspace of $E$ generated by $S$. Given a dual pair $\langle E, F\rangle$, we denote by $w(E, F)$ and $w(F, E)$ the induced weak topologies on $E$ and $F$. When $E=X$ is a Banach space and $F=X^{*}$ (its topological dual), we simply write $w=w\left(X, X^{*}\right)$ and $w^{*}=w\left(X^{*}, X\right)$. A locally convex space $E$ is said to have the Mazur property if every sequentially continuous element of $E^{\#}$ is continuous. A topological space $T$ is said to be FréchetUrysohn if, for each $B \subset T$, any element of $\bar{B}$ is the limit of a sequence contained in $B$. A subset $C$ of a topological space $T$ is said to be sequentially closed if no sequence in $C$ converges to a point in $T \backslash C$. Given a Banach space $X$, we write $B_{X}=\{x \in X:\|x\| \leq 1\}$ (the closed unit ball of $X$ ). A subspace $Y \subset X^{*}$ is said to be norming if the formula

$$
\left\||| x|\||=\sup \left\{x^{*}(x): x^{*} \in Y \cap B_{X^{*}}\right\}, \quad x \in X,\right.
$$


defines an equivalent norm on $X$. Given a compact topological space $K$, we denote by $C(K)$ the Banach space of all real-valued continuous functions on $K$, equipped with the supremum norm. For each $t \in K$, we write $\delta_{t} \in C(K)^{*}$ to denote the evaluation functional at $t$, i.e. $\delta_{t}(h):=h(t)$ for all $h \in C(K)$.

Throughout this paper $X$ is a Banach space. Given a $w^{*}$-dense subspace $Y \subset X^{*}$, we consider the subspace of $Y^{\#}$ defined by

$$
\widehat{X}_{Y}:=\left\{f \in Y^{\#}:\left.f\right|_{K} \text { is } w^{*} \text {-continuous for every } K \in \mathcal{K}(Y)\right\} .
$$

Note that $X$ can be identified with the subspace of $\widehat{X}_{Y}$ consisting of all $w^{*}$ continuous elements of $Y^{\#}$, that is, for any $w^{*}$-continuous $f \in Y^{\#}$ there is a unique $x \in X$ such that $\left\langle x, y^{*}\right\rangle=f\left(y^{*}\right)$ for all $y^{*} \in Y$. Observe that $\left\langle\widehat{X}_{Y}, Y\right\rangle$ is a dual pair and that an absolutely convex set $K \subset Y$ is $w^{*}$-compact if and only if it is $w\left(Y, \widehat{X}_{Y}\right)$-compact. In particular, the restriction of $\mu\left(\widehat{X}_{Y}, Y\right)$ to $X$ coincides with $\mu(X, Y)$. Grothendieck's characterization of the completion of a locally convex space (see e.g. [10, §21.9]), when applied to our setting, yields the following:

Fact 2.1. Let $Y \subset X^{*}$ be a $w^{*}$-dense subspace.

(i) $\left(\widehat{X}_{Y}, \mu\left(\widehat{X}_{Y}, Y\right)\right)$ is the completion of $(X, \mu(X, Y))$.

(ii) $(X, \mu(X, Y))$ is complete if and only if every element of $\widehat{X}_{Y}$ is $w^{*}$-continuous.

The following result is extracted from the proof of [9, Proposition 10]:

Fact 2.2. Let $Y \subset X^{*}$ be a $w^{*}$-dense and norm-closed subspace. Then

$$
\widehat{X}_{Y} \subset\left\{f \in Y^{\#}: f \text { is } w^{*} \text {-sequentially continuous }\right\} \subset Y^{*} .
$$

Thus, under the additional assumption that $Y$ is norm-closed, the Hahn-Banach theorem guarantees that for every $f \in \widehat{X}_{Y}$ there is some $x^{* *} \in X^{* *}$ such that $\left.x^{* *}\right|_{Y}=f$. Since $\left\{x^{* *} \in X^{* *}:\left.x^{* *}\right|_{Y}\right.$ is $w^{*}$-continuous $\}=X \oplus Y^{\perp}$, we get:

Fact 2.3. Let $Y \subset X^{*}$ be a $w^{*}$-dense and norm-closed subspace. Then $(X, \mu(X, Y))$ is complete if and only if

$$
\left\{x^{* *} \in X^{* *}:\left.x^{* *}\right|_{K} \text { is } w^{*} \text {-continuous for every } K \in \mathcal{K}(Y)\right\}=X \oplus Y^{\perp} .
$$

The following useful fact (see [9, Lemma 11]) will be needed several times.

Fact 2.4. Let $Y \subset X^{*}$ be a norming subspace. If $x^{* *} \in X^{* *} \backslash\left(X \oplus Y^{\perp}\right)$, then $Y \cap \operatorname{ker}\left(x^{* *}\right)$ is norming as well.

A general locally convex space very often lacks completeness but sometimes it satisfies a weaker property, called quasi-completeness, that is enough for major applications of completeness (Krein-Smulyan theorem, for instance). Recall that a locally convex space $E$ is said to be quasi-complete if every bounded and closed subset of $E$ is complete. We next show that in our setting quasi-completeness and completeness coincide.

Proposition 2.5. Let $Y \subset X^{*}$ be a $w^{*}$-dense and norm-closed subspace. Then $(X, \mu(X, Y))$ is quasi-complete if and only if it is complete. 
Proof. Take $A:=B_{X}$ and apply the bipolar theorem (see e.g. [ [6, Theorem 3.38]) in the dual pair $\left\langle\widehat{X}_{Y}, Y\right\rangle$ to obtain

$$
\bar{A}^{w\left(\widehat{X}_{Y}, Y\right)}=A^{\circ \circ}=\left\{f \in \widehat{X}_{Y}: f\left(y^{*}\right) \leq 1 \text { for all } y^{*} \in B_{Y}\right\} .
$$

Bearing in mind Mazur's theorem (see e.g. [6, Theorem 3.45]), we deduce that

$$
\bar{A}^{\mu\left(\widehat{X}_{Y}, Y\right)}=\bar{A}^{w\left(\widehat{X}_{Y}, Y\right)}=\left\{f \in \widehat{X}_{Y}: f\left(y^{*}\right) \leq 1 \text { for all } y^{*} \in B_{Y}\right\} .
$$

Suppose $(X, \mu(X, Y))$ is quasi-complete. We will show that $(X, \mu(X, Y))$ is complete by applying Fact 2.1. Take any $f \in \widehat{X}_{Y}$. Since $f \in Y^{*}$ (by Fact 2.2), we can assume that $f \in B_{Y^{*}}$ (normalize!). Then $f \in \bar{A}^{\mu\left(\widehat{X}_{Y}, Y\right)}$ (by (2.1)) and so there is a net $\left(x_{\alpha}\right)_{\alpha \in \Lambda}$ in $A$ which $\mu\left(\widehat{X}_{Y}, Y\right)$-converges to $f$. In particular, $\left(x_{\alpha}\right)_{\alpha \in \Lambda}$ is a bounded Cauchy net in the quasi-complete locally convex space $(X, \mu(X, Y))$. Then $\left(x_{\alpha}\right)_{\alpha \in \Lambda}$ is $\mu(X, Y)$-convergent to some $x \in X$ and so $f=x \in X$.

\section{Mazur PRoperty and Mackey COMPleteness}

The following proposition improves statement (b) in the introduction:

Proposition 3.1. Let $Y \subset X^{*}$ be a $w^{*}$-dense subspace such that:

(i) $(X, \mu(X, Y))$ is complete,

(ii) every $K \in \mathcal{K}(Y)$ has the following property: every convex $w^{*}$-sequentially closed subset of $K$ is $w^{*}$-closed.

Then $\left(Y, w^{*}\right)$ has the Mazur property.

Proof. Let $f: Y \rightarrow \mathbb{R}$ be linear and $w^{*}$-sequentially continuous. Since $(X, \mu(X, Y))$ is complete, in order to prove that $f$ is $w^{*}$-continuous it suffices to check that $\left.f\right|_{K}$ is $w^{*}$-continuous for any $K \in \mathcal{K}(Y)$ (Fact 2.1). Clearly, for a given $K \in \mathcal{K}(Y)$, the $w^{*}$-continuity of $\left.f\right|_{K}$ is equivalent to

$(\star) f^{-1}(C) \cap K$ is $w^{*}$-closed for every convex closed set $C \subset \mathbb{R}$.

Since $f$ is linear, for any convex $C \subset \mathbb{R}$ the set $f^{-1}(C) \cap K$ is convex and so it is $w^{*}$-closed if and only if it is $w^{*}$-sequentially closed (by (ii)). Therefore, the $w^{*}$-sequential continuity of $f$ ensures that $(\star)$ holds and the proof is finished.

Corollary 3.2. Suppose $X$ has property $\left(\mathcal{E}^{\prime}\right)$.

(i) Let $Y \subset X^{*}$ be a $w^{*}$-dense and norm-closed subspace. Then $(X, \mu(X, Y))$ is complete if and only if $\left(Y, w^{*}\right)$ has the Mazur property.

(ii) $X$ is fully Mackey complete if and only if it is fully Mazur.

The characterizations of fully Mazur and fully Mackey complete Banach spaces given in the next two theorems are our main results in this section.

Theorem 3.3. The following statements are equivalent:

(i) $X$ is fully Mazur.

(ii) $S_{1}(Y)=X^{*}$ for every norming and norm-closed subspace $Y \subset X^{*}$. 
Proof. (i) $\Rightarrow$ (ii) Suppose condition (ii) fails and fix a norming and norm-closed subspace $Z \subset X^{*}$ such that $X^{*} \backslash S_{1}(Z) \neq \emptyset$. Fix $x^{*} \in X^{*} \backslash S_{1}(Z)$, take the subspace $Y:=Z \oplus\left[x^{*}\right] \subset X^{*}$ (which is norming and norm-closed) and the functional $f \in Y^{*}$ defined by

$$
f\left(z^{*}+\lambda x^{*}\right):=\lambda \quad \text { for all } z^{*} \in Z \text { and } \lambda \in \mathbb{R} .
$$

Observe that $f$ is not $w^{*}$-continuous because $x^{*} \in X^{*}=\bar{Z}^{w^{*}}, f\left(x^{*}\right)=1$ and $\operatorname{ker}(f)=Z$.

Let us show that $f$ is $w^{*}$-sequentially continuous. Let $\left(y_{n}^{*}\right)_{n \in \mathbb{N}}$ be a sequence in $Y$ which $w^{*}$-converges to some $y^{*} \in Y$. Write

$$
y^{*}=z^{*}+\lambda x^{*} \quad \text { and } \quad y_{n}^{*}=z_{n}^{*}+\lambda_{n} x^{*}
$$

for some $z^{*}, z_{n}^{*} \in Z$ and $\lambda, \lambda_{n} \in \mathbb{R}$. Then

$$
\left(\left(z_{n}^{*}-z^{*}\right)+\left(\lambda_{n}-\lambda\right) x^{*}\right)_{n \in \mathbb{N}}
$$

is $w^{*}$-null. Since $z_{n}^{*}-z^{*} \in Z$ for all $n \in \mathbb{N}$ and $x^{*} \notin S_{1}(Z)$, we conclude that $f\left(y_{n}^{*}\right)=\lambda_{n} \rightarrow f\left(y^{*}\right)=\lambda$ as $n \rightarrow \infty$. This proves that $f$ is $w^{*}$-sequentially continuous. We have shown that $\left(Y, w^{*}\right)$ fails the Mazur property and therefore that $X$ is not fully Mazur.

(ii) $\Rightarrow$ (i) Let $Y \subset X^{*}$ be a norming and norm-closed subspace. To prove that $\left(Y, w^{*}\right)$ has the Mazur property, take a $w^{*}$-sequentially continuous $f \in Y^{\#}$. Since $f \in Y^{*}$ (Fact 2.2), there is $x^{* *} \in X^{* *}$ such that $\left.x^{* *}\right|_{Y}=f$. By contradiction, suppose $f$ is not $w^{*}$-continuous. Then $x^{* *} \in X^{* *} \backslash\left(X \oplus Y^{\perp}\right)$ and we can consider the norming and norm-closed subspace $Z:=Y \cap \operatorname{ker}\left(x^{* *}\right) \subset X^{*}$ (Fact 2.4). Condition (ii) applied to $Z$ ensures that $S_{1}(Z)=X^{*}$ and so the $w^{*}$-sequential continuity of $f$ implies that $Y=Z$, a contradiction which finishes the proof.

As an application, we generalize the result that Banach spaces having FréchetUrysohn dual ball are fully Mazur (see [9, Theorem 5]):

Corollary 3.4. If $X$ has Efremov's property $(\mathcal{E})$, then $S_{1}(Y)=X^{*}$ for every norming subspace $Y \subset X^{*}$. Consequently, $X$ is fully Mazur.

Proof. Let $Y \subset X^{*}$ be any norming subspace. By the Hahn-Banach separation theorem, we have $\overline{Y \cap B_{X^{*}}} w^{*} \supset \delta B_{X^{*}}$ for some $\delta>0$. On the other hand, $\overline{Y \cap B_{X^{*}}} w^{*}=S_{1}\left(Y \cap B_{X^{*}}\right)$ (since $X$ has property $(\mathcal{E})$ ) and therefore $S_{1}(Y)=X^{*}$. Theorem 3.3 now applies to deduce that $X$ is fully Mazur.

Theorem 3.5. $X$ is fully Mackey complete if and only if the following condition holds:

$(\mathcal{L K})$ For every norming and norm-closed subspace $Y \subset X^{*}$ and every $x^{*} \in X^{*} \backslash Y$ there is $K \in \mathcal{K}\left(X^{*}\right)$ such that $K \subset Y \oplus\left[x^{*}\right]$ and $x^{*} \in \overline{K \cap Y^{w^{*}}}$.

Proof. Suppose $X$ fails condition $(\mathcal{L K})$. Take a norming and norm-closed subspace $Y_{0} \subset X^{*}$ and $x^{*} \in X^{*} \backslash Y_{0}$ such that for every $K \in \mathcal{K}\left(X^{*}\right)$ we have

$$
x^{*} \in{\overline{K \cap Y_{0}}}^{w^{*}} \Longrightarrow K \nsubseteq Y_{0} \oplus\left[x^{*}\right]
$$


Set $Y:=Y_{0} \oplus\left[x^{*}\right]$ and define $f \in Y^{*}$ by declaring $f\left(y_{0}^{*}+\lambda x^{*}\right):=\lambda$ for every $y_{0}^{*} \in Y_{0}$ and $\lambda \in \mathbb{R}$. Note that $f$ is not $w^{*}$-continuous (because $x^{*} \in X^{*}={\overline{Y_{0}}}^{w^{*}}, f\left(x^{*}\right)=1$ and $\left.\operatorname{ker}(f)=Y_{0}\right)$. Thus, in order to prove that $(X,(\mu(X, Y))$ is not complete it is enough to show that $\left.f\right|_{K^{\prime}}$ is $w^{*}$-continuous for every $K^{\prime} \in \mathcal{K}(Y)$ (Fact 2.1).

By contradiction, suppose $\left.f\right|_{K^{\prime}}$ is not $w^{*}$-continuous. Then there is a net $\left(x_{\alpha}^{*}=y_{\alpha}^{*}+\lambda_{\alpha} x^{*}\right)_{\alpha \in \Lambda}$ in $K^{\prime}$ (where $y_{\alpha}^{*} \in Y_{0}$ and $\lambda_{\alpha} \in \mathbb{R}$ ) which $w^{*}$-converges to some $y^{*}+\lambda x^{*} \in K^{\prime}$ (where $y^{*} \in Y_{0}$ and $\lambda \in \mathbb{R}$ ) and such that $\left(\lambda_{\alpha}\right)_{\alpha \in \Lambda}$ does not converge to $\lambda$. Since $K^{\prime}$ is bounded, so is $\left(\lambda_{\alpha}\right)_{\alpha \in \Lambda}$. Fix $M>0$ such that $\left|\lambda_{\alpha}\right| \leq M$ for all $\alpha \in \Lambda$. By passing to a subnet if necessary we can assume that $\left(\lambda_{\alpha}\right)_{\alpha \in \Lambda}$ converges to some $\lambda^{\prime} \neq \lambda$. Set

$$
K:=\frac{K^{\prime}+M\left\|x^{*}\right\| B_{\left[x^{*}\right]}+\left\|y^{*}\right\| B_{\left[y^{*}\right]}}{\lambda-\lambda^{\prime}} \subset Y=Y_{0} \oplus\left[x^{*}\right]
$$

and notice that $K \in \mathcal{K}\left(X^{*}\right)$ since it is a sum of absolutely convex and $w^{*}$-compact sets. Moreover, the net

$$
\left(\frac{y_{\alpha}^{*}-y^{*}}{\lambda-\lambda^{\prime}}\right)_{\alpha \in \Lambda}=\left(\frac{x_{\alpha}^{*}-\lambda_{\alpha} x^{*}-y^{*}}{\lambda-\lambda^{\prime}}\right)_{\alpha \in \Lambda}
$$

is contained in $K \cap Y_{0}$ and $w^{*}$-converges to $x^{*}$, which contradicts (3.1). This shows that $(X,(\mu(X, Y))$ is not complete.

Conversely, we now prove that condition $(\mathcal{L K})$ implies that $X$ is fully Mackey complete. The argument is similar to the proof of (ii) $\Rightarrow$ (i) in Theorem 3.3, Let $Y \subset X^{*}$ be a norming and norm-closed subspace and take any $f \in \widehat{X}_{Y}$. Then $f \in Y^{*}$ (Fact 2.2) and so $f=\left.x^{* *}\right|_{Y}$ for some $x^{* *} \in X^{* *}$. If $f$ is not $w^{*}$-continuous, then $x^{* *} \notin X \oplus Y^{\perp}$ and therefore $Y_{0}:=Y \cap \operatorname{ker}\left(x^{* *}\right)=\operatorname{ker}(f)$ is norming (Fact 2.4). Pick $x^{*} \in Y \backslash Y_{0}$. Condition $(\mathcal{L K})$ applied to $Y_{0}$ and $x^{*}$ ensures the existence of $K \in \mathcal{K}\left(X^{*}\right)$ such that $K \subset Y_{0} \oplus\left[x^{*}\right] \subset Y$ and $x^{*} \in \overline{K \cap Y_{0}} w^{*}$. This contradicts the $w^{*}$-continuity of $\left.f\right|_{K}$, because $f$ vanishes on $K \cap Y_{0}$ and $f\left(x^{*}\right) \neq 0$. It follows that $f$ is $w^{*}$-continuous. This shows that $(X, \mu(X, Y))$ is complete (by Fact 2.1).

Corollary 3.6. If $X$ is fully Mazur (resp. fully Mackey complete), then any closed subspace of $X$ is fully Mazur (resp. fully Mackey complete).

Proof. Let $X_{0} \subset X$ be a a closed subspace and denote by $r: X^{*} \rightarrow X_{0}^{*}$ the bounded linear operator defined by $r\left(x^{*}\right):=\left.x^{*}\right|_{X_{0}}$ for every $x^{*} \in X^{*}$. Given any norm-closed subspace $Y_{0} \subset X_{0}^{*}$, the norm-closed subspace $Y:=r^{-1}\left(Y_{0}\right) \subset X^{*}$ is norming (for $X$ ) whenever $Y_{0}$ is norming (for $X_{0}$ ), see e.g. [6. p. 269, Exercise 5.6]. The conclusion now follows at once from Theorems 3.3 and 3.5 , bearing in mind the $w^{*}-w^{*}$-continuity of $r$.

\section{BANACH SPACES Which ARE NOT FUlly MACKEy COMPLETE}

The following technical result provides a sufficient condition on a Banach space to fail fully Mackey completeness. Recall that a topological space is said to be countably compact if every sequence in it has a cluster point.

Theorem 4.1. Let $T$ be a countably compact topological space with a distinguished point $\infty \in T$. Suppose there is a function $f: T \rightarrow X$ satisfying: 
(i) $f(\infty)=0$;

(ii) $Y:=\left\{x^{*} \in X^{*}: x^{*} \circ f\right.$ is continuous $\}$ is norming and norm-closed;

(iii) there exist $\varepsilon>0$ and $x_{\infty}^{*} \in X^{*}$ such that $D:=\left\{t \in T: x_{\infty}^{*}(f(t))>\varepsilon\right\}$ intersects every $\mathcal{G}_{\delta}$-set containing $\infty$.

Then $X$ is not fully Mackey complete.

Proof. By Theorem 3.5, it is enough to check that $X$ does not have property $(\mathcal{L K})$. Note that $x_{\infty}^{*} \circ f$ is not continuous at $\infty$ and so $x_{\infty}^{*} \notin Y$. Let $K$ be a bounded subset of $Y \oplus\left[x_{\infty}^{*}\right]$ with $x_{\infty}^{*} \in \overline{K \cap Y}^{w^{*}}$. We will prove that $\overline{K \cap Y}^{w^{*}}$ is not contained in $Y \oplus\left[x_{\infty}^{*}\right]$ and, therefore, $K$ is not $w^{*}$-compact.

To this end, we first construct by induction a sequence $\left(t_{n}\right)_{n \in \mathbb{N}}$ in $D$, a sequence $\left(x_{n}^{*}\right)_{n \in \mathbb{N}}$ in $K \cap Y$ and a decreasing sequence $\left(U_{n}\right)_{n \in \mathbb{N}}$ of open neighborhoods of $\infty$ such that, for each $n \in \mathbb{N}$, we have:

$\left(a_{n}\right) x_{n}^{*}\left(f\left(t_{j}\right)\right)>\varepsilon$ for every $j \leq n$;

$\left(b_{n}\right)\left|x_{n}^{*}(f(t))\right| \leq \frac{\varepsilon}{n}$ for every $t \in \overline{U_{n}}$;

$\left(c_{n}\right) t_{n} \in U_{n-1}$ (with the convention $U_{0}:=T$ ).

For the first step, take any $t_{1} \in D$. Since $x_{\infty}^{*} \in \overline{K \cap Y}^{w^{*}}$, we can pick $x_{1}^{*} \in K \cap Y$ such that $x_{1}^{*}\left(f\left(t_{1}\right)\right)>\varepsilon$. By the continuity of $x_{1}^{*} \circ f$, there is an open neighborhood $U_{1}$ of $\infty$ such that $\left|x_{1}^{*}(f(t))\right| \leq \varepsilon$ for every $t \in \overline{U_{1}}$. Suppose now that, for some $n \in \mathbb{N}$, we have already chosen $t_{1}, t_{2}, \ldots, t_{n} \in D, x_{1}^{*}, x_{2}^{*}, \ldots, x_{n}^{*} \in K \cap Y$ and $U_{1} \supset U_{2} \supset \cdots \supset U_{n}$ open neighborhoods of $\infty$ such that $\left(a_{i}\right),\left(b_{i}\right)$ and $\left(c_{i}\right)$ hold for every $i \leq n$. Pick an arbitrary $t_{n+1} \in D \cap U_{n}$ and choose $x_{n+1}^{*} \in K \cap Y$ with $x_{n+1}^{*}\left(f\left(t_{j}\right)\right)>\varepsilon$ for every $j \leq n+1$ (bear in mind that the $t_{i}$ 's belong to $D$ and $\left.x_{\infty}^{*} \in \overline{K \cap Y}^{w^{*}}\right)$. Now, the continuity of $x_{n+1}^{*} \circ f$ ensures the existence of an open neighborhood $U_{n+1}$ of $\infty$ contained in $U_{n}$ such that $\left|x_{n+1}^{*}(f(t))\right| \leq \frac{\varepsilon}{n+1}$ for every $t \in \overline{U_{n+1}}$. This finishes the inductive construction.

Let $x^{*} \in \overline{K \cap Y} w^{w^{*}}$ be any $w^{*}$-cluster point of the sequence $\left(x_{n}^{*}\right)_{n \in \mathbb{N}}$. Then

(a) $x^{*}\left(f\left(t_{n}\right)\right) \geq \varepsilon$ for every $n \in \mathbb{N}$;

(b) $x^{*}(f(t))=0$ for every $t \in \bigcap_{n \in \mathbb{N}} \overline{U_{n}}$.

We claim that $x^{*} \notin Y \oplus\left[x_{\infty}^{*}\right]$. Our proof is by contradiction. Suppose $x^{*}+\lambda x_{\infty}^{*} \in Y$ for some $\lambda \in \mathbb{R}$. By (a) and (b), $x^{*} \circ f$ is not continuous at any cluster point of the sequence $\left(t_{n}\right)_{n \in \mathbb{N}}$ (such cluster points exist since $T$ is countably compact and, by construction, they are contained in $\left.\bigcap_{n \in \mathbb{N}} \overline{U_{n}}\right)$, hence $\lambda \neq 0$. Observe that $\left|\left(x^{*}+\lambda x_{\infty}^{*}\right)(f(t))\right|>|\lambda| \varepsilon$ for every $t \in D \cap \bigcap_{n \in \mathbb{N}} U_{n}$. But $\infty \in \overline{D \cap \bigcap_{n \in \mathbb{N}} U_{n}}$ (because $D$ intersects every $\mathcal{G}_{\delta}$-set containing $\left.\infty\right)$ and therefore $\left(x^{*}+\lambda x_{\infty}^{*}\right) \circ f$ cannot be continuous at $\infty$, a contradiction.

The following corollary was already shown in $[8$, Corollary 5(ii)]. Here we prove it via the unifying approach of Theorem 4.1 .

Corollary 4.2. $\ell_{1}(\Gamma)$ is not fully Mackey complete whenever $\Gamma$ is uncountable.

Proof. Let $T:=\Gamma \cup\{\infty\}$ be the one-point compactification of the set $\Gamma$ equipped with the discrete topology. Define $f: T \rightarrow \ell_{1}(\Gamma)$ by declaring $f(\gamma):=e_{\gamma}$ for all $\gamma \in \Gamma$ and $f(\infty):=0$. Then $\left\{x^{*} \in \ell_{\infty}(\Gamma): x^{*} \circ f\right.$ is continuous $\}=c_{0}(\Gamma)$ is norming 
and norm-closed. Take $x_{\infty}^{*}:=\chi_{\Gamma} \in \ell_{\infty}(\Gamma)$ and fix any $0<\varepsilon<1$. Since $\Gamma$ is uncountable, $\{\infty\}$ is not a $\mathcal{G}_{\delta}$-set and so $\left\{t \in T: x_{\infty}^{*}(f(t))>\varepsilon\right\}=\Gamma$ intersects every $\mathcal{G}_{\delta}$-set containing $\infty$. The result now follows from Theorem 4.1

By putting together Corollaries 3.6 and 4.2, we get:

Corollary 4.3. If $X$ is fully Mackey complete, then it contains no subspace isomorphic to $\ell_{1}\left(\omega_{1}\right)$.

Corollary 4.4. $C\left(\left[0, \omega_{1}\right]\right)$ is not fully Mackey complete.

Proof. Let $T:=\left[0, \omega_{1}\right]$ and $\infty:=\omega_{1}$. Define $f: T \rightarrow C\left(\left[0, \omega_{1}\right]\right)$ by $f(\alpha):=\chi_{\left(\alpha, \omega_{1}\right]}$ for all $\alpha<\omega_{1}$ and $f(\infty):=0$. The subspace

$$
Y:=\left\{x^{*} \in C\left(\left[0, \omega_{1}\right]\right)^{*}: x^{*} \circ f \text { is continuous }\right\}
$$

is norm-closed (bear in mind that $f$ is bounded) and norming, because it contains the set $\left\{\delta_{\beta+1}: \beta<\omega_{1}\right\}$. Take $x_{\infty}^{*}:=\delta_{\omega_{1}}$ and fix any $0<\varepsilon<1$. Then the set $\left\{t \in T: x_{\infty}^{*}(f(t))>\varepsilon\right\}=\left[0, \omega_{1}\right)$ intersects every $\mathcal{G}_{\delta}$-set containing $\infty$ (since $\{\infty\}$ is not a $\mathcal{G}_{\delta}$-set). The result now follows from Theorem 4.1

We now focus on dual Banach spaces. Since $X$ is a norm-closed subspace of $X^{* *}$ which is norming for $X^{*}$, Theorem 3.3 implies that $X$ is $w^{*}$-sequentially dense in $X^{* *}$ whenever $X^{*}$ is fully Mazur. In fact, we have the following:

Theorem 4.5. If $X^{*}$ is fully Mackey complete, then $X$ is $w^{*}$-sequentially dense in $X^{* *}$.

Proof. By Corollary 4.3. $X^{*}$ contains no subspace isomorphic to $\ell_{1}\left(\omega_{1}\right)$, which implies that $X$ contains no subspace isomorphic to $\ell_{1}$ (see e.g. [17, Proposition 4.2]).

Fix $x^{* *} \in X^{* *} \backslash X$. Since $X$ is a norming (for $X^{*}$ ) and norm-closed subspace of $X^{* *}$, Theorem 3.5 ensures the existence of $K \in \mathcal{K}\left(X^{* *}\right)$ such that $K \subset X \oplus\left[x^{* *}\right]$ and $x^{* *} \in \overline{K \cap X}^{w^{*}}$. This implies that $K \cap X$ is not weakly compact and therefore it is not weakly sequentially compact (due to the Eberlein-Šmulian theorem). Take any sequence $\left(x_{n}\right)_{n \in \mathbb{N}}$ in $K \cap X$ without weakly convergent subsequences. Since $X$ does not contain subspaces isomorphic to $\ell_{1}$, we can suppose without loss of generality that $\left(x_{n}\right)_{n \in \mathbb{N}}$ is weakly Cauchy, thanks to Rosenthal's $\ell_{1}$-theorem (see e.g. [17, Proposition 4.2]). Therefore, $\left(x_{n}\right)_{n \in \mathbb{N}}$ is $w^{*}$-convergent to an element of $X^{* *}$ of the form $x+\lambda x^{* *}$ with $x \in X$ and $\lambda \neq 0$ (since $K \subset X \oplus\left[x^{* *}\right]$ ). Thus, $\left(\frac{x_{n}-x}{\lambda}\right)_{n \in \mathbb{N}}$ is a sequence in $X$ which $w^{*}$-converges to $x^{* *}$. This shows that $X$ is $w^{*}$-sequentially dense in $X^{* *}$.

Banach spaces which are $w^{*}$-sequentially dense in their bidual have been widely studied in the literature. We next include some related remarks on fully Mackey complete dual spaces which follow from Theorem 4.5.

Remark 4.6. Every $w^{*}$-sequentially continuous linear functional $f: X^{* *} \rightarrow \mathbb{R}$ is norm-continuous when restricted to $X$, i.e. $\left.f\right|_{X} \in X^{*}$. Therefore, the equality $f\left(x^{* *}\right)=\left\langle x^{* *},\left.f\right|_{X}\right\rangle$ holds for every $x^{* *} \in X^{* *}$ which is the $w^{*}$-limit of a sequence contained in $X$. It follows that if $X$ is $w^{*}$-sequentially dense in $X^{* *}$, then $\left(X^{* *}, w^{*}\right)$ 
has the Mazur property. This provides new non-trivial examples of Banach spaces which are not fully Mackey complete, such as the long James space $J\left(\omega_{1}\right)$ (see [5]). Indeed, $J\left(\omega_{1}\right)$ is the dual of a Banach space $X$ which is not $w^{*}$-sequentially dense in $X^{* *}$, since $\left(J\left(\omega_{1}\right)^{*}, w^{*}\right)$ fails the Mazur property.

Remark 4.7. If $X$ is $w^{*}$-sequentially dense in $X^{* *}$, then $X$ contains no subspace isomorphic to $\ell_{1}$ (see e.g. [16, Proposition 3.9]) and, moreover, in each of the following particular cases $\left(B_{X^{* *}}, w^{*}\right)$ is Fréchet-Urysohn:

- $X$ is separable, by the Odell-Rosenthal and Bourgain-Fremlin-Talagrand theorems (see e.g. [17, Theorem 4.1]).

- $X$ is Asplund, in fact, in this case $X^{*}$ is weakly Lindelof determined, i.e. $\left(B_{X^{* *}}, w^{*}\right)$ is Corson (see [4, Theorem III-4] and [13, Corollary 8]).

As a consequence:

Corollary 4.8. Suppose $X$ is separable. Then $X^{*}$ is fully Mackey complete if and only if $\left(B_{X^{* *}}, w^{*}\right)$ is Fréchet-Urysohn if and only if $X$ contains no subspace isomorphic to $\ell_{1}$.

Corollary 4.9. Suppose $X$ is Asplund. Then $X^{*}$ is fully Mackey complete if and only if $X^{*}$ is weakly Lindelof determined.

By an almost disjoint family we mean an infinite family of pairwise almost disjoint infinite subsets of $\mathbb{N}$, where two sets are said to be almost disjoint if they have finite intersection. For any almost disjoint family $\mathcal{F}$, we denote by $K_{\mathcal{F}}$ the Stone (compact topological) space associated to the Boolean algebra generated by $\mathcal{F}$ and the finite subsets of $\mathbb{N}$. Notice that there is a natural decomposition

$$
K_{\mathcal{F}}=\mathbb{N} \cup\left\{u_{N}: N \in \mathcal{F}\right\} \cup\{\infty\},
$$

where each point of $\mathbb{N}$ is isolated, the basic open neighborhoods of each $u_{N}$ are of the form $\left\{u_{N}\right\} \cup(N \backslash F)$ where $F \subset \mathbb{N}$ is finite, and the basic open neighborhoods of $\infty$ are of the form $K_{\mathcal{F}} \backslash \bigcup_{N \in \mathcal{F}_{0}}\left(\left\{u_{N}\right\} \cup N\right)$ where $\mathcal{F}_{0} \subset \mathcal{F}$ is finite. Then $K_{\mathcal{F}}$ is scattered (of height 3 ) and so

$$
C\left(K_{\mathcal{F}}\right)^{*}=\ell_{1}\left(K_{\mathcal{F}}\right)=\ell_{1}(\mathbb{N}) \oplus \ell_{1}(\mathcal{F}) \oplus\left[\delta_{\infty}\right] .
$$

Observe that $\ell_{1}(\mathbb{N})$ is a norming (since $\mathbb{N}$ is dense in $K_{\mathcal{F}}$ ) and norm-closed subspace of $C\left(K_{\mathcal{F}}\right)^{*}$. Under the Continuum Hypothesis, the construction in [2, Section 4] provides a maximal (with respect to inclusion) almost disjoint family $\mathcal{F}$ for which no sequence in the convex hull of $\left\{\delta_{n}: n \in \mathbb{N}\right\}$ is $w^{*}$-convergent to $\delta_{\infty}$. We will improve such construction as follows:

Theorem 4.10. Under the Continuum Hypothesis, there exists a maximal almost disjoint family $\mathcal{F}$ such that:

(i) No sequence in $\ell_{1}(\mathbb{N})$ is $w^{*}$-convergent to $\delta_{\infty}$.

(ii) $C\left(K_{\mathcal{F}}\right)$ is not fully Mackey complete.

Part (i) will be proved with the help of Lemmas 4.11 and 4.12 below. The first one is a refinement of [2, Lemma 4.2]: 
Lemma 4.11. Let $\mathcal{F}=\left\{N_{r}: r \in \mathbb{N}\right\}$ be a countable almost disjoint family and let $\left(\lambda_{i, j}\right)_{i, j \in \mathbb{N}} \in \mathbb{R}^{\mathbb{N} \times \mathbb{N}}$ be a matrix satisfying the following properties:

(i) $\lim _{i \rightarrow \infty} \lambda_{i, j}=0$ for every $j \in \mathbb{N}$;

(ii) $\sum_{j \in \mathbb{N}}\left|\lambda_{i, j}\right|<\infty$ for every $i \in \mathbb{N}$;

(iii) $\lim _{i \rightarrow \infty} \sum_{j \in \mathbb{N}} \lambda_{i, j}=1$.

If

$$
\lim _{i \rightarrow \infty} \sum_{j \in N_{1} \cup \ldots \cup N_{r}} \lambda_{i, j}=0 \quad \text { for every } r \in \mathbb{N},
$$

then there exists an infinite set $N^{\prime} \subset \mathbb{N}$ such that $\mathcal{F} \cup\left\{N^{\prime}\right\}$ is almost disjoint and

$$
\limsup _{i \rightarrow \infty} \sum_{j \in N^{\prime}} \lambda_{i, j} \geq \frac{1}{2}
$$

Proof. Write $\tilde{N}_{r}:=N_{1} \cup \ldots \cup N_{r}$ for all $r \in \mathbb{N}$. We will construct by induction a sequence $\left(F_{r}\right)_{r \in \mathbb{N}}$ of finite subsets of $\mathbb{N}$ and a strictly increasing sequence $\left(n_{r}\right)_{r \in \mathbb{N}}$ of natural numbers as follows. Take any finite set $F_{1} \subset \mathbb{N}$ and any $n_{1} \in \mathbb{N}$. Given $r \in \mathbb{N}, r \geq 2$, suppose the finite sets $F_{1}, \ldots, F_{r-1} \subset \mathbb{N}$ and $n_{1}<\cdots<n_{r-1}$ in $\mathbb{N}$ have already been chosen. By (4.1) and (iii), we can find $n_{r} \in \mathbb{N}$ with $n_{r}>n_{r-1}$ in such a way that

(a) $\sum_{j \in \tilde{N}_{r}} \lambda_{n_{r}, j} \leq \frac{1}{16}$;

(b) $\sum_{j \in \mathbb{N}} \lambda_{n_{r}, j} \geq \frac{3}{4}$.

Since $F_{1} \cup \ldots \cup F_{r-1}$ is finite, (i) allows us to assume further that

(c) $\sum_{j \in F_{1} \cup \ldots \cup F_{r-1}}\left|\lambda_{n_{r}, j}\right| \leq \frac{1}{16}$.

By (ii), there is a finite set $F_{r} \subset \mathbb{N} \backslash \tilde{N}_{r}$ satisfying

$$
\sum_{j \in \mathbb{N} \backslash\left(\tilde{N}_{r} \cup F_{r}\right)}\left|\lambda_{n_{r}, j}\right| \leq \frac{1}{16} .
$$

Notice that (a), (b) and (4.2) yield

$$
\sum_{j \in F_{r}} \lambda_{n_{r}, j}=\sum_{j \in \mathbb{N}} \lambda_{n_{r}, j}-\sum_{j \in \tilde{N}_{r}} \lambda_{n_{r}, j}-\sum_{j \in \mathbb{N} \backslash\left(\tilde{N}_{r} \cup F_{r}\right)} \lambda_{n_{r}, j} \geq \frac{3}{4}-\frac{1}{16}-\frac{1}{16}=\frac{5}{8} .
$$

This finishes the inductive construction.

Let us check that $N^{\prime}:=\bigcup_{r \in \mathbb{N}} F_{r}$ satisfies the required properties. On one hand, for each $r \in \mathbb{N}$ with $r \geq 2$ we have

$$
N^{\prime} \backslash F_{r} \subset\left(F_{1} \cup \ldots \cup F_{r-1}\right) \cup\left(\mathbb{N} \backslash\left(\tilde{N}_{r} \cup F_{r}\right)\right),
$$

hence

$$
\sum_{j \in N^{\prime} \backslash F_{r}} \lambda_{n_{r}, j}+\sum_{j \in F_{1} \cup \ldots \cup F_{r-1}}\left|\lambda_{n_{r}, j}\right|+\sum_{j \in \mathbb{N} \backslash\left(\tilde{N}_{r} \cup F_{r}\right)}\left|\lambda_{n_{r}, j}\right| \geq 0 .
$$


This inequality, (c), (4.2) and (4.3) yield

$$
\begin{aligned}
& \sum_{j \in N^{\prime}} \lambda_{n_{r}, j}=\sum_{j \in F_{r}} \lambda_{n_{r}, j}+\sum_{j \in N^{\prime} \backslash F_{r}} \lambda_{n_{r}, j} \\
& \geq \sum_{j \in F_{r}} \lambda_{n_{r}, j}-\sum_{j \in F_{1} \cup \ldots \cup F_{r-1}}\left|\lambda_{n_{r}, j}\right|-\sum_{j \in \mathbb{N} \backslash\left(\tilde{N}_{r} \cup F_{r}\right)}\left|\lambda_{n_{r}, j}\right| \geq \frac{5}{8}-\frac{1}{16}-\frac{1}{16}=\frac{1}{2} .
\end{aligned}
$$

As $r \in \mathbb{N}$ is arbitrary, it follows that

$$
\limsup _{i \rightarrow \infty} \sum_{j \in N^{\prime}} \lambda_{i, j} \geq \frac{1}{2}
$$

On the other hand, (i) ensures that $\lim _{i \rightarrow \infty} \sum_{j \in F} \lambda_{i, j}=0$ whenever $F \subset \mathbb{N}$ is finite, therefore $N^{\prime}$ is infinite. By construction, $N^{\prime} \cap N_{1} \subseteq F_{1}$ and for each $r \in \mathbb{N}$ with $r \geq 2$ the intersection $N^{\prime} \cap N_{r}$ is contained in the finite set $F_{1} \cup F_{2} \cup \ldots \cup F_{r-1}$. This shows that $\mathcal{F} \cup\left\{N^{\prime}\right\}$ is an almost disjoint family.

Lemma 4.12. Let $\left\{\left(\lambda_{i, j}^{\alpha}\right)_{i, j \in \mathbb{N}}: \alpha<\omega_{1}\right\}$ be a family of matrices of $\mathbb{R}^{\mathbb{N} \times \mathbb{N}}$ satisfying properties (i), (ii) and (iii) of Lemma 4.11. Then there exists an almost disjoint family $\mathcal{F}$ such that for every $\alpha<\omega_{1}$ there is $N_{\alpha}^{\prime} \in \mathcal{F}$ for which the sequence $\left(\sum_{j \in N_{\alpha}^{\prime}} \lambda_{i, j}^{\alpha}\right)_{i \in \mathbb{N}}$ does not converge to 0 .

Proof. Let $\mathcal{G}=\left\{N_{r}: r \in \mathbb{N}\right\}$ be any countable almost disjoint family. If there is $r \in \mathbb{N}$ for which $\left(\sum_{j \in N_{r}} \lambda_{i, j}^{0}\right)_{i \in \mathbb{N}}$ does not converge to 0 , then we set $\mathcal{F}_{0}:=\mathcal{G}$ and $N_{0}^{\prime}:=N_{r}$. Otherwise, $\lim _{i \rightarrow \infty} \sum_{j \in N_{r}} \lambda_{i, j}^{0}=0$ for all $r \in \mathbb{N}$. Observe that for each $r \in \mathbb{N}$ with $r \geq 2$ we have

$$
\sum_{j \in N_{1} \cup \ldots \cup N_{r}} \lambda_{i, j}^{0}=\sum_{j \in N_{1} \cup \ldots \cup N_{r-1}} \lambda_{i, j}^{0}+\sum_{j \in N_{r}} \lambda_{i, j}^{0}-\sum_{j \in N_{r} \cap\left(N_{1} \cup \ldots \cup N_{r-1}\right)} \lambda_{i, j}^{0}
$$

for all $i \in \mathbb{N}$ and

$$
\lim _{i \rightarrow \infty} \sum_{j \in N_{r} \cap\left(N_{1} \cup \ldots \cup N_{r-1}\right)} \lambda_{i, j}^{0}=0,
$$

by property (i) and the finiteness of $N_{r} \cap\left(N_{1} \cup \ldots \cup N_{r-1}\right)$. This clearly implies (by induction on $r$ ) that

$$
\lim _{i \rightarrow \infty} \sum_{j \in N_{1} \cup \ldots \cup N_{r}} \lambda_{i, j}^{0}=0 \quad \text { for every } r \in \mathbb{N},
$$

so Lemma 4.11 can be applied to find an infinite set $N_{0}^{\prime} \subset \mathbb{N}$ for which $\mathcal{F}_{0}:=\mathcal{G} \cup\left\{N_{0}^{\prime}\right\}$ is an almost disjoint family and $\left(\sum_{j \in N_{0}^{\prime}} \lambda_{i, j}^{0}\right)_{i \in \mathbb{N}}$ does not converge to 0 .

We now construct, by transfinite induction on $\alpha<\omega_{1}$, an increasing chain $\left(\mathcal{F}_{\alpha}\right)_{\alpha<\omega_{1}}$ of countable almost disjoint families and sets $N_{\alpha}^{\prime} \in \mathcal{F}_{\alpha}$ for which the sequence $\left(\sum_{j \in N_{\alpha}^{\prime}} \lambda_{i, j}^{\alpha}\right)_{i \in \mathbb{N}}$ does not converge to 0 . Suppose that $0<\alpha<\omega_{1}$ and that $\mathcal{F}_{\beta}$ and $N_{\beta}^{\prime}$ are already constructed for every $\beta<\alpha$. If

$$
\lim _{i \rightarrow \infty} \sum_{j \in N} \lambda_{i, j}^{\alpha}=0 \quad \text { for every } N \in \bigcup_{\beta<\alpha} \mathcal{F}_{\beta},
$$


then we can apply the argument above to $\bigcup_{\beta<\alpha} \mathcal{F}_{\beta}$ (which is a countable almost disjoint family) and the matrix $\left(\lambda_{i, j}^{\alpha}\right)_{i, j \in \mathbb{N}}$ in order to get an infinite set $N_{\alpha}^{\prime} \subset \mathbb{N}$ such that $\mathcal{F}_{\alpha}:=\left(\bigcup_{\beta<\alpha} \mathcal{F}_{\beta}\right) \cup\left\{N_{\alpha}^{\prime}\right\}$ is almost disjoint and $\left(\sum_{j \in N_{\alpha}^{\prime}} \lambda_{i, j}^{\alpha}\right)_{i \in \mathbb{N}}$ does not converge to 0 . Otherwise, if (4.4) fails, then we take $\mathcal{F}_{\alpha}:=\bigcup_{\beta<\alpha} \mathcal{F}_{\beta}$ and any $N_{\alpha}^{\prime} \in \mathcal{F}_{\alpha}$ witnessing the failure of (4.4).

Clearly, $\mathcal{F}:=\bigcup_{\beta<\omega_{1}} \mathcal{F}_{\beta}$ is an almost disjoint family satisfying the required property.

Proof of Theorem 4.10. (i) The set of all matrices of $\mathbb{R}^{\mathbb{N} \times \mathbb{N}}$ satisfying properties (i), (ii) and (iii) of Lemma 4.11 has cardinality $\mathfrak{c}$ and so, under the Continuum Hypothesis, it can be enumerated as $\left\{\left(\lambda_{i, j}^{\alpha}\right)_{i, j \in \mathbb{N}}: \alpha<\omega_{1}\right\}$. Let $\mathcal{F}$ be the almost disjoint family given by Lemma 4.12. To check that $\mathcal{F}$ is maximal, take any infinite set $N=\left\{n_{k}: k \in \mathbb{N}\right\} \subset \mathbb{N}$ and define a matrix $\left(\lambda_{i, j}\right)_{i, j \in \mathbb{N}} \in \mathbb{R}^{\mathbb{N} \times \mathbb{N}}$ by declaring $\lambda_{i, j}:=1$ if $n_{i}=j$ and $\lambda_{i, j}:=0$ otherwise. Obviously, it satisfies properties (i), (ii) and (iii) of Lemma 4.11, hence there is $N^{\prime} \in \mathcal{F}$ such that $\left(\sum_{j \in N^{\prime}} \lambda_{i, j}\right)_{i \in \mathbb{N}}$ does not converge to 0 , which clearly implies that $N \cap N^{\prime}$ is infinite. This shows that $\mathcal{F}$ is maximal.

Suppose $\left(x_{i}^{*}\right)_{i \in \mathbb{N}}$ is a sequence in $\ell_{1}(\mathbb{N})$ which $w^{*}$-converges to $\delta_{\infty}$ in $C\left(K_{\mathcal{F}}\right)^{*}$. For each $i \in \mathbb{N}$ we write $x_{i}^{*}=\sum_{j \in \mathbb{N}} \lambda_{i, j} \delta_{j}$, where $\lambda_{i, j} \in \mathbb{R}$ and $\sum_{j \in \mathbb{N}}\left|\lambda_{i, j}\right|<\infty$. Then

$$
\lim _{i \rightarrow \infty} \lambda_{i, j}=\lim _{i \rightarrow \infty} x_{i}^{*}\left(\chi_{\{j\}}\right)=\delta_{\infty}\left(\chi_{\{j\}}\right)=0 \quad \text { for every } j \in \mathbb{N}
$$

and

$$
\lim _{i \rightarrow \infty} \sum_{j \in \mathbb{N}} \lambda_{i, j}=\lim _{i \rightarrow \infty} x_{i}^{*}\left(\chi_{K_{\mathcal{F}}}\right)=\delta_{\infty}\left(\chi_{K_{\mathcal{F}}}\right)=1 .
$$

Therefore, $\left(\lambda_{i, j}\right)_{i, j \in \mathbb{N}}=\left(\lambda_{i, j}^{\alpha}\right)_{i, j \in \mathbb{N}}$ for some $\alpha<\omega_{1}$. But then there exists $N_{\alpha}^{\prime} \in \mathcal{F}$ such that $\left(\sum_{j \in N_{\alpha}^{\prime}} \lambda_{i, j}\right)_{i \in \mathbb{N}}$ does not converge to 0 , which is a contradiction since

$$
\sum_{j \in N_{\alpha}^{\prime}} \lambda_{i, j}=x_{i}^{*}\left(\chi_{\left\{u_{N_{\alpha}^{\prime}}\right\} \cup N_{\alpha}^{\prime}}\right) \quad \text { for all } i \in \mathbb{N}
$$

and $\delta_{\infty}\left(\chi_{\left\{u_{N_{\alpha}^{\prime}}\right\} \cup N_{\alpha}^{\prime}}\right)=0$.

(ii) The space $C\left(K_{\mathcal{F}}\right)$ is not fully Mazur by (i) and Theorem 3.3. On the other hand, since $K_{\mathcal{F}}$ is scattered of countable height, $\left(B_{C\left(K_{\mathcal{F}}\right)^{*}}, w^{*}\right)$ is sequential, meaning that every $w^{*}$-sequentially closed subset of $B_{C\left(K_{\mathcal{F}}\right)^{*}}$ is $w^{*}$-closed (see [12, Theorem 3.2]). Hence $C\left(K_{\mathcal{F}}\right)$ has property $\left(\mathcal{E}^{\prime}\right)$ and so $C\left(K_{\mathcal{F}}\right)$ is not fully Mackey complete (apply Corollary 3.2). The proof is finished.

We finish the paper with some open questions:

Problem 4.13. Are fully Mazur and fully Mackey completeness equivalent?

As we pointed out in Corollary 3.2. Problem 4.13 has an affirmative answer for Banach spaces with property $\left(\mathcal{E}^{\prime}\right)$.

Problem 4.14. Does fully Mackey completeness imply property $\left(\mathcal{E}^{\prime}\right)$ or the weaker Corson's property $(\mathcal{C})$ ? 
Problem 4.15. Is $X^{*}$ fully Mackey complete whenever $X$ is $w^{*}$-sequentially dense in $X^{* *}$ ?

Remark 4.7 makes clear that a negative answer to Problem 4.15 would be based on a non-separable and non-Asplund space $X$ without subspaces isomorphic to $\ell_{1}$.

Acknowledgements. The authors wish to thank A. Avilés for valuable discussions on the topic of this paper. A.J. Guirao was supported by projects MTM2017-83262C2-1-P (AEI/FEDER, UE) and 19368/PI/14 (Fundación Séneca). G. MartínezCervantes and J. Rodríguez were supported by projects MTM2014-54182-P and MTM2017-86182-P (AEI/FEDER, UE) and 19275/PI/14 (Fundación Séneca).

\section{REFERENCES}

[1] G. Androulakis and M. Ziemke, The closedness of the generator of a semigroup, Semigroup Forum 93 (2016), no. 3, 589-606.

[2] A. Avilés, G. Martínez-Cervantes, and J. Rodríguez, Weak*-sequential properties of JohnsonLindenstrauss spaces, J. Funct. Anal. (2018), https://doi.org/10.1016/j.jfa.2018.09.007

[3] J. Bonet and B. Cascales, Noncomplete Mackey topologies on Banach spaces, Bull. Aust. Math. Soc. 81 (2010), no. 3, 409-413.

[4] R. Deville and G. Godefroy, Some applications of projective resolutions of identity, Proc. London Math. Soc. (3) 67 (1993), no. 1, 183-199.

[5] G. A. Edgar, A long James space, Measure theory, Oberwolfach 1979 (Proc. Conf., Oberwolfach, 1979), Lecture Notes in Math., vol. 794, Springer, Berlin, 1980, pp. 31-37.

[6] M. Fabian, P. Habala, P. Hájek, V. Montesinos, and V. Zizler, Banach space theory. The basis for linear and nonlinear analysis, CMS Books in Mathematics/Ouvrages de Mathématiques de la SMC, Springer, New York, 2011.

[7] A. S. Granero and M. Sánchez, The class of universally Krein-Šmulian Banach spaces, Bull. Lond. Math. Soc. 39 (2007), no. 4, 529-540.

[8] A. J. Guirao and V. Montesinos, Completeness in the Mackey topology, Funct. Anal. Appl. 49 (2015), no. 2, 97-105, Translation of Funktsional. Anal. i Prilozhen. 49 (2015), no. 2, $21-33$.

[9] A. J. Guirao, V. Montesinos, and V. Zizler, A note on Mackey topologies on Banach spaces, J. Math. Anal. Appl. 445 (2017), no. 1, 944-952.

[10] G. Köthe, Topological vector spaces. I, Translated from the German by D. J. H. Garling. Die Grundlehren der mathematischen Wissenschaften, Band 159, Springer-Verlag New York Inc., New York, 1969.

[11] M. Kunze, A Pettis-type integral and applications to transition semigroups, Czechoslovak Math. J. 61(136) (2011), no. 2, 437-459.

[12] G. Martínez-Cervantes, Banach spaces with weak*-sequential dual ball, Proc. Amer. Math. Soc. 146 (2018), no. 4, 1825-1832.

[13] J. Orihuela, On weakly Lindelöf Banach spaces, Progress in functional analysis (Peñíscola, 1990), North-Holland Math. Stud., vol. 170, North-Holland, Amsterdam, 1992, pp. 279-291.

[14] A. Plichko, Three sequential properties of dual Banach spaces in the weak* topology, Topology Appl. 190 (2015), 93-98.

[15] J. Rodríguez, On integration in Banach spaces and total sets, to appear in Quaest. Math.

[16] J. Rodríguez and G. Vera, Uniqueness of measure extensions in Banach spaces, Studia Math. 175 (2006), no. 2, 139-155.

[17] D. van Dulst, Characterizations of Banach spaces not containing $l^{1}$, CWI Tract, vol. 59, Centrum voor Wiskunde en Informatica, Amsterdam, 1989. 
Instituto Universitario de Matemática Pura y Aplicada, Universitat Politècnica de València, Camino de Vera s/n, 46022 Valencia, Spain

E-mail address: anguisa2@mat.upv.es

Dpto. de Matemáticas, Facultad de Matemáticas, Universidad de Murcia, 30100 EsPINARDO (MURCIA), SPAIN

E-mail address: gonzalomartinezcervantes@gmail.com

Dpto. de Ingeniería y Tecnología de Computadores, Facultad de Informática, Universidad de Murcia, 30100 Espinardo (Murcia), Spain

E-mail address: joserr@um.es 